\title{
A Woman-in-Effect
}

\author{
Claudine Potvin
}

In a dry run at sketching herself into urban space, marking a terrain, shaking up the streets, the narrator-heroine moves through the city with a slow, arduous step. An eleven year-old girl working the street in the middle of winter is at the centre of this text: circling back, for the millionth time, to the same spot on the sidewalk, rounding the same corner, waiting for a car to interrupt her endless course. What interrupts her course, however, is the narrator's periodic entrance on this stage in self-reflexive commentaries which point to the fantasmatic nature of an account of prostitution projected from a warm cafe, a perfumed bath, a cozy bed. The prostitute without a name, continually chased off the sidewalk and into dark corners, already represents herself as object of derision for the benefit of polite society. Within this overdetermined space of representation, the constitutive illusion of strutting along the sidewalk with this girl is a risky identification which places the "other" on the feminist agenda of writing (in) the city.

"She" is created from the close explorations of semantic chains whose elements have changed textual as well as conceptual positions, at least in terms of valorization: from time to space, the same to other, paranoia to hysteria, city to labyrinth, mastery to nonmastery, truth to fiction. (Alice A. Jardine, Gynesis)

Entre le travail et l'ascenseur, entre la chambre et le théâtre, seule la marche, lente et pénible, heureuse parfois, inscrit la narratrice dans la ligne urbaine, dans la pensée, dans l'ouverture, dans le désir. Le cheminement de l'hérö̈ne/narratrice dessine le visible dans la cité, la marque d'un contour, une forme de reconnaissance. Pistes d'essai, à l'essai. Soulever la rue. Le texte citadin s'enchaîne, se déchaîne. Effets de drogues hallucinogènes. 
Pas de jeu possible pour la petite fille qui se prostitue. A woman-ineffect. Une faible audace sur les joues mal fardées et sur les lèvres rouge sang ouvertes sur le regard enlevant des passants. La petite fille fait les cent pas, les mille pas tracés au crayon noir sur la carte de la cité. Se balader, avoir l'air de chercher son mec, s'allumer une cigarette, désinvolte, attacher les cordons défaits de sa botte, profiter del'éclairage pour étaler la marchandise, tourner le coin sans conviction, répéter les gestes d'une chasse où on tue l'animal à coup portant, à coups de piastres. La petite fille se relève toujours comme après les scènes érotiques d'un film porno. C'est une vieille petite fille au sourire fatigué (France Théoret, Nécessairement putain). La blessure un fake intérieur le corps se multiplie se déplace se schize se déchire sous la pression des doigts invisibles. Le coeur se soulève.

Elle a perdu son père un matin de mai. N'a jamais su s'il fallait en être attristée. Est venue à la ville parce qu'il n'y avait pas d'autre lieu. La ville absorbe, gobe, fascine, nourrit un peu, permet, s'avance au-devant des femmes qui s'y donnent. La ville, magique, lourde sous la pluie, frileuse l'hiver. ... entre l'horreur et l'utopie, entre l'exaltation et la désintégration. La ville au crépuscule est la polis des hommes. La ville, via l'image, via les corps comme un calcul mental (Nicole Brossard, "Pré(e)").

Elle a commencé à travailler en décembre, juste avant les vacances scolaires, et il faisait si froid que son étroite jupe de cuir lui formait une carapace métallique autour des cuisses laissant une sensation désagréable d'emprisonnement. Une de ses copines lui avait raconté qu'elle s'en sortirait mieux dans la grande ville, qu'il y avait là de nombreuses avenues et tant de mystères à explorer.

Elle revoyait ce morceau de trottoir pour la millième fois, elle si jeune en cette fin de siècle. Chaque soir, il lui ramenait de nouveaux visages, souriant au même passage, exigeant la même rançon. Se pourrait-il que tous les trottoirs mènent à Rome les trottoirs abîmés de toutes ces filles qui courent les rues? L'enflure de sa cheville gonfle les veines bleues de givre. Elle est la marche même d'une femme enfant haute et délestée de toute épaisseur (France Théoret, Nécessairement putain). L'éclatement semble si proche se résorbe sous les coups la chair crue des femmes offerte en sacrifice même les jours de sabbat se tapisse se (dé)couvre de néons bariolés se déguise, vulgaire dit-on, marquée au fer, prisonnière.

Asphyxie relais délai provoquer le jour faire fondre la neige égarée sur les épaules la petite fille ne réfléchit pas à / ne se réfléchit pas dans les 
flaques la slush couvre les souliers délicats la petite fille ne se pose pas de questions au sujet de ... la ville enchante les passants la petite fille ne souffre pas, pas encore, gelée, elle se tient droite, haut(e) perchée, attirée par les escaliers qui s'enfilent comme des salles de musée.

Elle n'a jamais visité de musée. Ni lu ni parlé d'art. Seul son corps s'expose sur la voie publique événement en soi performance de balcon matériau brute que la narratrice s'entête à pétrir argile de feu figurine smoke-fired juste à temps pour que se dégage le noir de la pierre l'ocre de la terre le gris des blocs de ciment clay/clé sans serrure serrer de plus près la matière obscure des villes enceintes avortées coupées de leurs verdures ou de leurs femmes façonner des sexes-modèles primitive-fired.

Dans son rêve, est absorbée par un tapis roulant circule d'un terminal à l'autre en transit l'aéroport gronde les passagers en spirale elle refuse le cri sans bagages sans organes elle n'est pas toute/à elle déplacement du trottoir neutralité soudaine la simulation s'attarde sur la rampe noire la nuit contient son poids de fuite sortir du rêve de l'autre débarquer amorcer sa marche elle se réveille au moment précis de la panne de courant.

Dans sa vie, Gerry fait figure de maître. Pour le pimp en transition, rien n'est important. Il loue des vidéos tous les soirs, des films pour adultes, où d'horribles vieilles dames enculent des petites filles. Il faut être un homme pour imaginer de tels scénarios, pense la petite fille. Effets de perversion. La pornographie REGARDE les femmes puisqu'elles sont l'objet de la représentation pornographique. La petite fille regarde Gerry intérioriser le vidéo. Une maîtresse d'école violée par une classe de petits morveux. Sa vision des choses l'étouffe. Gerry est presque tendre vicieux indifférent à la tristesse des femmes. On ne peut pas aimer Gerry. On vità ses côtés sans savoir pourquoi pour la maigreur de son corps la faiblesse de ses poings sans doute la couleur de ses yeux l'épaisseur de son regard. Le front de Gerry lisse luisant large comme une balançoire contient toutes les lignes de tous les hommes crispés sur son corps de petite fille. Dans la chambre d'hôtel, fixe les rides accrochées à la lumière sèche insolente de la nuit. Le lampadaire envahit le matelas découvert et mou. Un sexe étranger s'agite fébrilement transpire. Une araignée arpente le mur de gauche tourne le regard du côté du vide. Elle aimerait se laisser glisser dans les bras de Gerry une fois pour toutes. 
Ma vision d'une petite fille en train de faire les cent pas sur un trottoir en attendant patiemment que le feu tourne au rouge et qu'un véhicule interrompe apparemment sa course pour l'interpeller et l'enlever temporairement au langage d'une rue qui ne lui appartiendra jamais demeure biaisée par le préjugé de celle qui se situe toujours ailleurs dans les cafés dans les chambres chaudes et lumineuses de sa Rue des Érables dans les duvets blancs et moelleux dans le fantasme de la prostitution et de l'usage des femmes dans les enfants douillets des belles familles qu'elle n'a jamais vraiment connues dans la projection d'un vécu imaginé dans le cinéma cérébral de son adolescence dans les bains parfumés dans les bulles de plaisir teintées de bleu poudre dans l'écriture (les apparences entre elle et moi se situent au ras du désir piégé ou de l'illusion).

Le plaisir du trottoir, c'est les autres filles. Pour elles le trottoir la marche ne prennent leur sens que dans l'apparat le revêtement le rire amer la complicité l'angoisse habillée de rouge de noir de blanc de rose la peur en talons hauts talons aiguilles pour se piquer d'en savoir plus que les autres. Rose, Janet, Julie, Michelle, Wendy, noms collés aux fesses anonymes de la rue, sans famille. Tout ce qui leur reste, un nom premier inventé pour la circonstance plus authentique que le nom de baptême nom de combat la sentence des hommes ne leur doit rien l'identité d'une putain ne se mesure pas au revenu ni au nombre de clients une fois suffit pour perdre son nom.

La ville ne les nomme plus elle les regarde passer identiques surfaites toujours sommée de les déloger de la rue Sanguinet des chasses gardées du Parc Lafontaine des hôtels luxueux de Vancouver de la troisième avenue d'un village perdu au milieu des prairies des prudes quartiers de Toronto la pure l'amour puritain en circuit fermé l'éros en vitrine sur les écrans permissifs de Mexico et de Guadalajara. On astique les trottoirs et on remet ça ailleurs.

La femme/narratrice/hérö̈ne s'éparpille dans la ville, semble-t-il, observant les limites et les frontières de l'autre, l'autre vision, l'autre versant, jaugeant l'absence de son oeil et de sa vue dans les traces d'un jeu de pieds dans la neige. Écrire (dans) la ville, se pencher sur soi, sur le réel. S'asseoir dans la ville, assiéger l'asphalte, s'inventer des pistes vierges, se fabriquer une métropole. MONOPOLE. De femmes. 
Elle accumule des pas inutiles peu payants la chair est fraîche la conviction manque la rue offre davantage, elle le sent elle pourrait choisir de s'arrêter elle pourrait magasiner elle aussi. Elle avait lu dans un journal qu'une prostituée de Floride, lesbienne de surcroît, disait l'article, avait assasiné cinq hommes et qu'on allait l'exécuter. Le premier lui avait versé de la térébenthine dans l'anus, brûlé le cul effrontément, et elle ne l'avait pas toléré. Les clients en demandent toujours plus la putain se représente elle-même en objet de dérision en "WOMAN $\mathrm{ZOO}^{\prime}$. Porno ( du grec porne), prostitution, et graphie: dessin, écriture (représentation). Collaboration / prostitution is at the very basis of what is deemed 'obscene' or indecent in polite society. (Susanne Kappeler, The Pornography of Representation)

La petite fille n'avait jamais rien entendu de pareil, n'avait jamais senti le vent la frôler. La peur se glissait en elle tel un serpent s'enroulant autour de la taille lui enrobant les seins d'écailles transparentes s'effilochant au bout des ongles.

L'étreinte d'un serpent amer fausse la sexualité des petites filles ne laisse pas monter le jus bloque la jouissance avant même que ne se forme une vulve dorée une moule à l'odeur saline la petite fille n'a. jamais mangé de fruits de mer ne connaît pas ces restaurants fabuleux que fréquente la narratrice sa gastronomie se limite à la saveur des autres. L'anorexique se nourrit mal.

Ne se souvient de rien a tout effacé les points les pauses les interrogations surtout ne voit pas l'utilité de chercher à comprendre vit le moment le mot instantané le cliché l'indifférence des lieux le mépris de celles qui traversent en diagonale le paysage elle marche et autour ça passe dans la rue pleine des quatre heures de l'après-midi rue Saint-Laurent (France Théoret, Nécessairement putain) le sable n'existe plus au coin de la rue seuls les détours se placent dans la ligne géométrique de ce quadrilatère circonscrit circulez sens unique débarassez le terrain clairez la place évacuez sentir que toute une ville d'hommes vous veut et ne vous veut pas en même temps le désir tout à côté de la faute chasser l'objet pour ne pas se punir soi-même se cacher les faits la rue que l'on fabrique agir en flics policer le corps des femmes et des petites filles exclure civiliser ranger se servir/de ailleurs autrement abuser s'abuser à quoi bon la ville interprète étrangement le territoire des femmes le leur retire les confine à des sous-sols encombrés de mannequins de pièces mécaniques de bras arrachés de doigts tordus de maux de tête de mutilations les limite à 
mille plateaux sans échelle à des chambres solitaires à des appareils stériles.

Pourvu qu'elle marche la petite fille sait qu'elle s'approprie tout au moins le pas la dép(f)ense elle dépasse telle une bête délimite son espace arrose le sol l'imprègne de l'odeur des femmes flouées trop tôt par les circonstances en ville les hommes puent différemment leurs gros corps suent se morfondent au travail sur les plis du cou un relent de bière pisse comme entre les seins de Julie.

Faire couler de l'encre entre les fentes des édifices, occuper les lieux, inscrire le trottoir à la limite du corps des autres, oublier les ruelles de l'enfance, traverser la frontière. La narratrice interroge la fiction et se demande s'il faut imaginer un destin en dehors de la marche pour la petite fille qui fait le trottoir à onze ans. L'objectif se situant sans doute dans le déplacement... refuse d'attendre que le corps bouge en dehors de l'écran remet la pellicule reprend le film élimine la séquence urbaine déplace l'imagination pornographique de ses personnages.

Les chemins sont glissants. Surveiller les blocs de glace risquer la fracture la fêlure des os du coeur sentir le sang bouillir dans les veines se retourner sur leur passage ni sable ni sel le vice (se) paie. Comme il est creux ce coeur dans la poitrine de la ville (Clarice Lispector, "Le manifeste de la ville," Où étais-tupendant la nuit?). Il sait depuis toujours que la marche ne mène qu'à la reconnaissance de la nuit. 